\title{
Estudio preliminar sobre la comunidad de primates en el bosque protector del Oglán Alto. Arajuno-Pastaza
}

\author{
Gabriel Alberto Carrillo Bilbao ${ }^{1,2,3,4}$ \& Sarah Martin-Solano ${ }^{1,5}$ \\ ${ }^{1}$ Estación Científica Amazónica de la Universidad Central del Ecuador, Quito, Ecuador. \\ gacarrillo@andinanet.net \\ ${ }^{2}$ Escuela de Biología y Química, Universidad Central del Ecuador, Quito, Ecuador. \\ ${ }^{3}$ Universidad Internacional Menéndez Pelayo, Madrid, España. \\ ${ }^{4}$ Ecociencia, Quito, Ecuador. \\ ${ }^{5}$ Unidad de Biología del comportamiento, Universidad de Lieja, Lieja, Bélgica. \\ sarahmartinsolano@gmail.com
}

Recibido: 2010-05-27, aprobado: 2010-08-31

\begin{abstract}
RESUMEN.- Los primates juegan un papel ecológico crucial en los ecosistemas donde se encuentran, por lo tanto los esfuerzos que se realicen para su conservación apoyarán de manera directa la preservación de otros elementos del mismo ecosistema. La Reserva Amazónica del Oglán Alto es, en su mayoría, un bosque primario piemontano y de tierras bajas, entre 420 y 1.100 m.s.n.m., de donde se registran ocho especies de primates, de las cuales cinco se encuentran en el Libro Rojo (1) y además cuatro están bajo presión de caza. Por tal razón, es imprescindible conocer el estado real de la comunidad de primates presentes en esta reserva. Gracias a la elaboración de un sistema de senderos y a la creación de tres transectos, se registró seis especies de primates de las ocho mencionadas en el plan de manejo. Los índices de diversidad sugerirían una diversidad considerable. En el Ecuador, además, existen muy pocos estudios de primates en bosques de estribaciones amazónicas, por lo que estos resultados aportarán datos a las comparaciones y correlaciones tanto ecológicas como etológicas con estudios de primates de amazonía baja y de occidente.
\end{abstract}

PALABRAS CLAVE: Amazonía, diversidad, ecología, Oglan Alto, primates.

ABSTRACT.- Primates play a crucial ecological role within the ecosystems they inhabit; therefore any effort done in favor of their conservation, will also support 
the preservation of the other elements of their ecosystem. The Amazonian Reserve of Oglán Alto is in its majority, a lowland forest and a piemontano primary forest, between 420 and 1100 m.s.n.m. Eight species of primates are registered, five of them are in the Red Book of Ecuador (1) and other four are under hunting pressure which makes it critical to know the real status of the primate community in this reserve. Thanks to the creation of a trail system and three transects, we have registered six of the eight registered species according with the environmental management plan from the Reserve. The diversity index suggests a considerable diversity. Considering that up to date, there is little data recorded in this region, these results will contribute to comparative research, ecological as well as ethological studies, on primates from the low Amazonia and the West.

KEY WORDS: Amazon, diversity, ecology, Oglan Alto, primates.

\section{INTRODUCCIÓN}

La Amazonía, considerada el bosque tropical más extenso del planeta, abarca alrededor de 6,6 millones de kilómetros cuadrados, lo que representa el $53 \%$ de las selvas tropicales (2). Los bosques amazónicos se extienden en nueve países: Ecuador, Brasil, Surinam, Guyana, Guayana Francesa, Venezuela, Perú, Bolivia, y Colombia, presentando altos niveles de diversidad y endemismo (2) y la más alta diversidad de primates en el mundo, siendo así que por lo menos seis nuevas especies de primates se han descubierto en los diez últimos años (3) .

A partir del análisis de los rangos de distribución de primates, se ha dividido la Amazonía en cuatro áreas o distritos de endemismo (Guayana, Ecuador, Perú y Brazil). Esto, a su vez, ha sido validado por estudios en otros grupos de vertebrados y por el análisis de los rangos de distribución de primates $(4$, $5,6,7,8,9,10,11)$. El distrito de Ecuador se encuentra dividido en dos zonas, Imeri y Napo que al igual que la de Guiana, mantienen más del $40 \%$ del total de su extensión en áreas protegidas (12).

Las actividades antropogénicas son importantes en la pérdida de hábitats en la Amazonía ecuatoriana, especialmente por la conversión de bosques en tierras dedicadas a la agricultura, ganadería o industria $(13,14)$. En el caso de los primates, la destrucción de sus hábitats, la caza y la captura de ejemplares vivos para su exportación y uso local son las mayores amenazas para su conservación $(15,16)$. Como producto de la explosión demográfica, que coincidencialmente es mayor en países tropicales, se sabe que entre 1985 y 1995 América Latina y el Caribe han perdido el $9,7 \%$ de bosques. 
En concreto, los países en los que se registran poblaciones de primates, perdieron $125140 \mathrm{~km}^{2}$ de bosques anualmente, lo cual no sólo afecta a los propios primates, sino a todos los componentes del ecosistema $(16,14)$. Por otro lado, el avance del desarrollo social, petrolero y minero, la creación de proyectos de construcción de carreteras, colonización, proyectos hidroeléctricos y tala de bosques, entre otros, han causado que el Ecuador pierda anualmente alrededor del 1,8\% de su cobertura boscosa original (14). Por tanto, priorizar en el desarrollo e implementación de planes efectivos de conservación es fundamental para detener la fragmentación de estos bosques (12).

El Ecuador, hasta la fecha, registra 20 especies de primates distribuidas en cuatro familias: Cebidae, Atelidae, Pitheciidae y Aotidae (17, 1, 18). Un total de 15 especies se encuentran en los bosques tropicales de la Amazonía ecuatoriana.

Existe poca información acerca de los aspectos ecológicos y etológicos de muchas de las especies de primates presentes en el país $(19,20)$. Prueba de esto es que la mayoría de los recursos bibliográficos existentes sobre los primates ecuatorianos está basada en fuentes extranjeras (14); por tal razón, es necesario obtener datos relevantes sobre el estado actual de las poblaciones de primates (21), tomando en cuen- ta que el Ecuador registra el $40 \%$ del total de especies de primates del Nuevo Mundo (Platyrrhini) y el 10,7 \% del total de especies a nivel mundial.

Existen numerosas razones por las cuales se deben realizar esfuerzos en la conservación de los primates. Los primates juegan un papel ecológico crucial en los ecosistemas donde estos se encuentran, y su pérdida causaría dramáticos efectos; por ejemplo, todas las especies de primates forman parte de las cadenas alimenticias actuando como presas o predadores y son también dispersores de semillas. Para tener una idea de la importancia que tienen los primates como dispersores en los bosques tropicales, más del $90 \%$ de las plantas leñosas dependen de especies frugívoras para dispersar sus semillas, y los primates comprenden la mayor porción del total de la biomasa de los frugívoros neotropicales $(22,23,17,16$, 24, 25). Dada la diversidad de primates en el Ecuador, su importancia en la dinámica de los bosques tropicales amazónicos es alta y su ausencia causaría un efecto cascada en la dinámica de los ecosistemas (26). Recordemos que usualmente tienen bajas tasas de crecimiento, una reproducción con largos intervalos entre nacimientos y un tiempo largo de desarrollo (27), lo cual indica que sus bajas densidades poblacionales en bosques intervenidos o en peligro los hacen indicadores muy sensibles del estrés antropogénico (21). 
Finalmente, las similitudes genéticas y ecológicas entre los primates no humanos y los humanos pueden permitir dar paso a la necesidad instintiva y ética de conservar y preservar los hábitats de nuestros parientes cercanos (25). Por lo tanto, los esfuerzos satisfactorios que se realicen para su conservación apoyarán de manera directa a la preservación de otros elementos del mismo ecosistema (24).

\section{Antecedentes}

La Reserva Amazónica del Oglán Alto es un área de 3344 ha ubicada en las coordenadas $0^{\circ} 5^{\prime} 19,9^{\prime \prime} \mathrm{N}$ - $80^{\circ} 30^{\prime} 46,0^{\prime} \mathrm{O}$, entre los 420 y 1.000 m.s.n.m. en el Cantón Arajuno, Provincia de Pastaza. En su mayoría es bosque primario piemontano y de tierras bajas según la clasificación vegetal de Sierra (28). Estos bosques reciben una pluviosidad de $2.000-4.000 \mathrm{~mm}$ anuales y no se registran más de cuatro meses consecutivos con una pluviosidad menor a los $100 \mathrm{~mm}$. Este bosque se caracteriza por grandes árboles, llegando en algunos casos a cincuenta o sesenta metros $(1,20,29,30)$.

El tipo de ecosistema presente en la reserva se extiende a lo largo de la línea ecuatorial, en las áreas con tierras bajas. La gran diversidad de especies vegetales es otra característica de estos bosques; de 400 individuos muestreados se han registrado 200 especies de árboles en 128 géneros y 49 familias (31). Ade- más, la gran variedad de especies vegetales no arbóreas son las que conforman la mayor porción de la biomasa vegetal, lo cual es un verdadero potencial alimenticio para las distintas especies que se desarrollan en este tipo de ecosistemas (32).

El plan de manejo ambiental de la Reserva, realizado previamente a este proyecto (29), fue la base para la decisión de estudiar las poblaciones de primates, ya que se registraron ocho especies: Callithrix pygmaea, Saguinus fuscicollis, Cebus albifrons, Saimiri sciureus, Lagothrix poeppigii, Ateles belzebuth, Alouatta seniculus y Pithecia monachus, de las cuales cinco (Lagothrix poeppigii, Ateles belzebuth, Alouatta seniculus, Cebus albifrons y Saguinus fuscicollis) se encuentran incluidas en el libro rojo de mamíferos ecuatorianos (1) y cuatro (Alouatta seniculus, Ateles belzebuth, Lagothrix poeppigii y Cebus albifrons) se consideran como especies sometidas a presiones de caza según Redford y Robinson (33). Por tal razón fue imprescindible conocer el estado real de la comunidad de primates presentes en esta reserva. En abril de 2006 se inició el Proyecto Primates Oglán con una salida de prospección. En julio de 2006 se realizó la socialización del Proyecto frente a representantes de ACIA (Asociación de Comunidades Indígenas Arajuno), logrando la aceptación por parte de las comunidades para la ejecución del proyecto. 


\section{MÉTODOS}

Este estudio se llevó a cabo desde enero del 2007 a noviembre del 2008, para lo cual se destinaron diez días de cada mes para la apertura y mapeo de senderos y transectos y diez días para la búsqueda, habituación y recolección de datos.

Se establecieron tres transectos: uno de tres kilómetros, otro de 1,5 km y otro de $300 \mathrm{~m}$. Además de los transectos, se construyó una red de senderos de alrededor de $17 \mathrm{~km}$, los cuales abarcan el área de una manera espaciada y heterogénea evitando así el re-conteo de individuos (Figura 1).
Cabe recalcar que este estudio se apoya en la metodología de "Roadside counts" (34) para monitorear siguiendo un sistema de senderos, el cual utiliza zonas heterogéneas donde los senderos mantienen distancias óptimas de separación para evitar el reconteo de individuos. Para minimizar el sesgo en las observaciones se tomó muy en cuenta el protocolo del método de "Traversing the area and detecting animals" (35) y "Line Transect Sampling from a Curving Path" (36) que básicamente es similar al de "Roadside counts", pero pone énfasis en variables como condiciones del hábitat respecto a variacio-

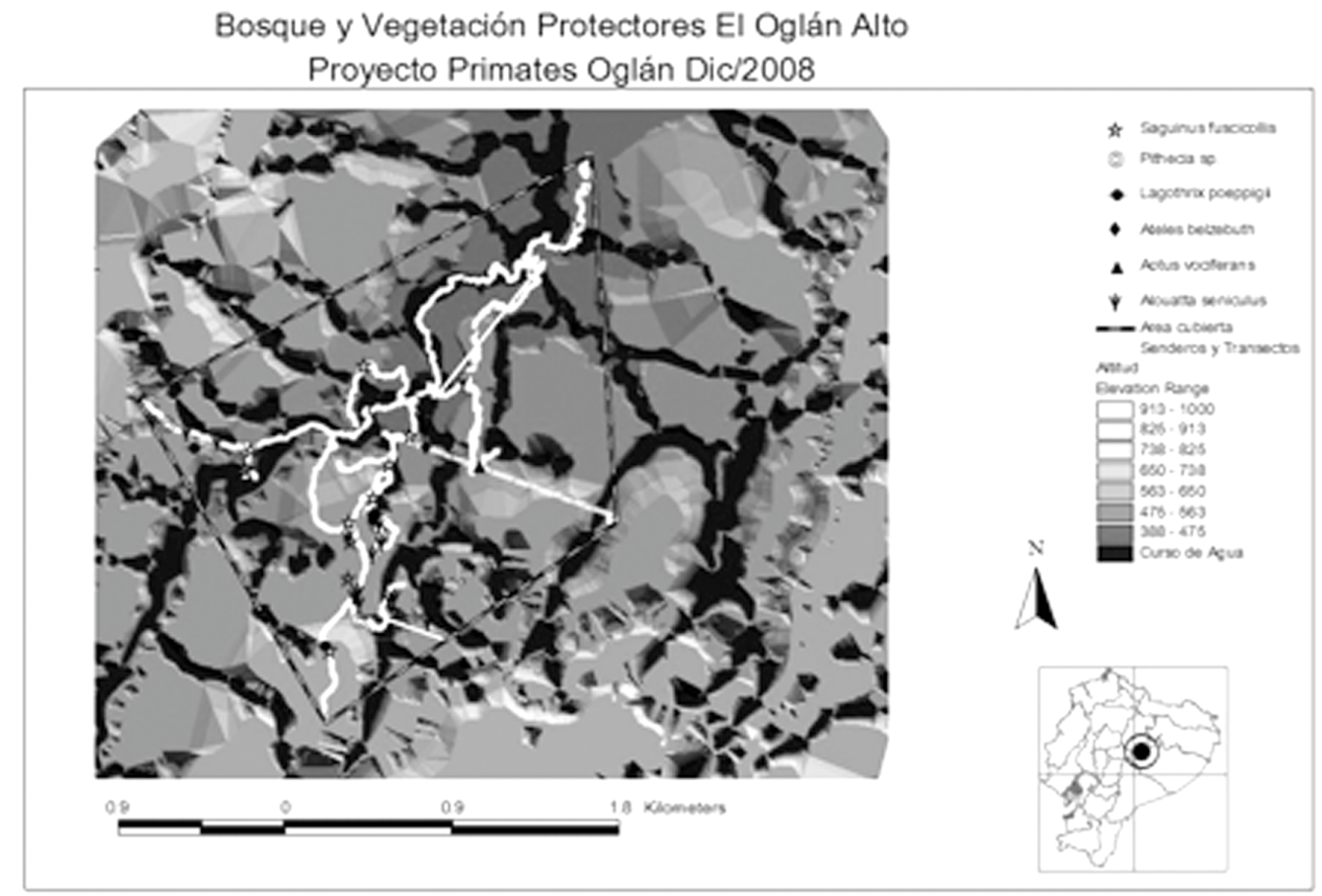

Figura 1. Avistamientos, transectos y senderos del Proyecto Primates Oglán 
nes estacionales y condiciones climáticas. Por la dificultad propia asociada a las características geográficas de la zona las metodologías tradicionales requieren más tiempo y esfuerzo para su construcción.

Los primeros diez días de cada mes, dos grupos de observación (un investigador y un asistente) recorrieron los senderos y los transectos en un sentido entre las 6:00 h y 11:00 h y en el sentido contrario entre 13:00 h y 18:00 h, abarcando los picos de actividad de los primates; la velocidad promedio fue de $1,2 \mathrm{~km} / \mathrm{h}(36,35)$ con paradas cortas cada $100 \mathrm{~m}$. Se determinó la presencia de primates mediante rastros (fecas), audición y visualización por medio de binoculares $8 \mathrm{x}$ $40(37,38)$.

Se tomaron datos de ubicación de todos los registros y el número total de individuos observados; se siguió la misma metodología de mapeo de senderos y transectos para marcar y graficar estos puntos en un mapa. Se usaron planillas de avistaje para el registro de datos del estudio de monitoreo que incluyeron: ubicación, fecha, hora de inicio y hora de finalización de la observación, especie, número de individuos, composición grupal y datos descriptivos como observaciones y eventos inusuales.

El área de vida de los grupos estudiados fue realizada mediante el programa Arcview 3.2 con la extensión
Animal Movement 2.04 beta. Se generó un "minumun convex poligon" que es el polígono que incluye a todas las observaciones de menor área y se calculó la superficie del polígono (20, $39,40)$.

El mapeo de los senderos y de los transectos se realizó con brújula y flexómetro; los senderos fueron marcados cada $25 \mathrm{~m}$. Los datos de dirección y distancia fueron transformados a coordenadas UTMs usando un punto georeferenciado con un GPS y trigonometría básica en hojas de cálculo de Microsoft Excel; finalmente los mapas fueron elaborados en los programas Arcview 3.2 y Arcgis 9.0. Se han incluido en el mapa también caminos vecinales y árboles marcados así como los puntos de avistaje de primates (Figura 1).

Los índices de diversidad fueron calculados de acuerdo con Jost (41, 42, 43). Para la estimación del número de especies esperadas se utilizaron los índices Chaol y Chaol-bc.

Los paquetes estadísticos utilizados para todos los análisis estadísticos fueron ESTIMATES, que se usó para estimar el número de especies totales, SPSS para los cálculos de la curva acumulativa, SPADE en el que se generaron los análisis de Chaol y Chao1-bc; y DiversityCalculatorLJost500sp para calcular los índices de diversidad. 


\section{RESULTADOS}

Seis especies de primates se han registrado en el área de estudio: tres correspondientes a la familia Atelidae y una especie de cada una de las familias Pitheciidae, Aotidae y Cebidae. De las seis especies se obtuvieron 42 registros: Alouatta seniculus (20 registros), Saguinus fuscicollis (15 registros), Ateles belzebuth (un registro), Aotus vociferans (un registro), Lagothrix poeppigii (dos registros) y Pithecia sp.(tres registros) mediante el método de observación directa, auditiva (Figura 1).

Se registraron seis grupos de $A$. seniculus (21 individuos), cuatro grupos de $S$. fuscicollis (16 individuos), un grupo de L. poeppigii ( $\geq 35$ individuos), dos grupos de Pithecia sp. ( $\geq 2$ individuos), un grupo de Ateles belzebuth ( $\geq$ 8 individuos) y un grupo de Aotus vociferans ( $\geq 1$ individuo; Tabla 1 ).

El grupo A de $A$. seniculus está constituido por un macho adulto, una hembra adulta, un juvenil y un infante independiente; adicionalmente, se re- gistró un individuo macho adulto solitario. El grupo A de $S$. fuscicollis está constituido $\geq 5$ individuos siendo todos sus integrantes de similar tamaño y se han categorizado como adultos o subadultos. El área de vida preliminar para el grupo A de $A$. seniculus fue de 26,6 ha y para el grupo A de $S$. fuscicollis fue de 46,8 hectáreas (Figura 2).

Un total de 807 minutos de observación de primates se contabilizó hasta la fecha: 588 para Alouatta, 109 para Saguinus, 62 para Lagothrix, 8 para Aotus, 10 para Pithecia y 30 para Ateles. (Tabla 2).

La curva de acumulación de especies (Figura 3) muestra que la pendiente aún se encuentra en ascenso, no se evidencia una tendencia a estabilizarse.

El índice de Diversidad Exponencial de Shannon (effective number of species) es de 4,046. El índice Inverso de Simpson (Diversity of order ) es de 3,460. El valor del índice de Chaol (especies esperadas) es de 10,5 y del índice del Chao1-bc es de 7,5.

Tabla 1. Avistajes de Especies según el Número de grupos (n.o de Grupos), solitario (ind/solitarios), media de individuos por grupo (Ind/solitarios) y total de individuos (Total ind), en la reserva del Oglan Alto.

\begin{tabular}{lcccc}
\hline Especie & $\begin{array}{c}\text { n.o de } \\
\text { Grupos }\end{array}$ & Ind/solitarios & $\begin{array}{c}\text { n.o } \\
\text { ind/grupo }\end{array}$ & Total ind \\
\hline Alouatta seniculus & 6 & 1 & 3.5 & 21 \\
Saguinus fuscicollis & 4 & 0 & 4 & 16 \\
Pithecia sp. & 2 &. & $\geq 1$ & $\geq 2$ \\
Ateles belzebuth & 1 & 0 & $\geq 8$ & $\geq 8$ \\
Aotus vociferans & 1 &. & $\geq 1$ & $\geq 1$ \\
Lagothrix poeppigii & 1 & 0 & $\geq 35$ & $\geq 35$ \\
\hline
\end{tabular}




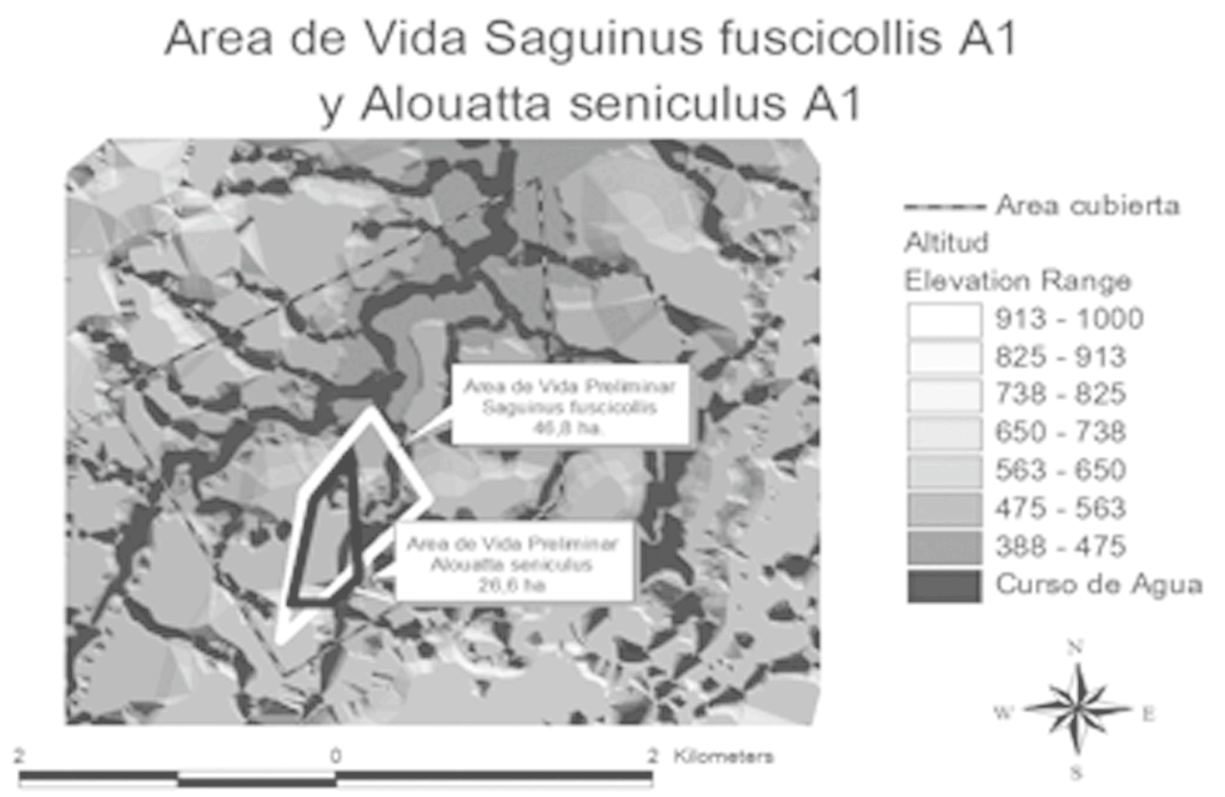

Figura 2. Área de vida de Alouatta seniculus A1 y de Saguinus fuscicollis A1

\section{DISCUSIÓN Y}

\section{CONCLUSIONES}

Mediante la metodología planteada se han observado seis especies de primates, lo que difiere con respecto a los datos incluidos en el plan de manejo de la reserva, en el que se incluyeron ocho especies, a partir de las encuestas realizadas. Hay que destacar que las estimaciones de la riqueza de especies alcanzan diez (Chao1) y 7,5 (Chao1bc) especies en la zona de estudio. Adicionalmente, basados en información bibliográfica sobre el área de vida de las distintas especies $(44,45,46$, $47,40)$, se podría sugerir que hasta la fecha se han registrado un total de 15 grupos de primates, datos que serán aceptados o rechazados a medida que se continúe con la toma de datos demográficos. La curva acumulativa de especies no se saturó con los datos recogidos (Figura 3), lo que sugiere que el monitoreo tiene que continuar. Estos análisis permitirán en el futuro realizar y justificar comparaciones con análisis de datos de un monitoreo a largo plazo o con estudios en otras zonas.

Los valores obtenidos con los diferentes índices podrían sugerir que existe una diversidad considerable, lo que podría ser producto de las características de la reserva. El número de especies registradas mediante métodos 
Tabla 2. Comportamiento por minutos registrados

\begin{tabular}{llc}
\hline Especie & Comportamiento & Minutos Registrados \\
\hline Alouatta seniculus & Vter & 140 \\
& Vxx,Val,Esc & 448 \\
Saguinus fuscicollis & Vxx,Val,Esc & 108 \\
& Vcon & 1 \\
Lagothrix poeppigii & Vxx,Esc,Mxx & 62 \\
Ateles belzebuth & Mxx & 30 \\
Pithecia sp. & Esc,Val & 5 \\
Aotus vociferans & NR & 5 \\
& Vter & 8 \\
\hline
\end{tabular}

Vter=vocalización territorial, $\mathrm{Vxx}=$ Vocalización desconocida, Val=vocalización alarma, Esc=escape, $\mathrm{Mxx}=$ Movimiento desconocido, $\mathrm{Nr}=$ no registra

directos e indirectos para este estudio es de seis especies de primates, siendo mayor al número de especies registradas en los estudios llevados a cabo en el Parque Nacional Napo-Galeras y en el Parque Nacional Sangay con tres y ninguna especie respectivamente (48, 49). Al contar con escasos sitios de comparación en amazonía de estribaciones, podemos comparar con otros sitios de amazonía baja. Por ejemplo, en un estudio realizado por Heymann y Encarnación (50), encontraron en un monitoreo realizado en el río Curaray y Napo de tres a cinco especies; sin embargo, hay que tomar en cuenta que estos monitoreos se realizaron a corto plazo (menos de un mes). En el Parque Nacional Yasuní, los estudios a largo plazo han logrado identificar diez especies de primates $(51,52)$, lo cual reflejaría la importancia de realizar monitoreos constantes y a largo plazo, minimizando el uso de registros bibliográficos ya que esto no permite evidenciar de manera concreta la presencia o ausencia de primates de un sector.

En el futuro se monitorearán zonas más bajas para abarcar la totalidad del rango altitudinal de la reserva y hacer comparaciones a lo largo de este gradiente. Se predice que el número de especie aumentará a medida que se incluyan más zonas de tierras bajas.

Esta reserva representa un refugio para la fauna ya que, en las zonas aledañas, los primates y demás mesomamíferos sufren una alta presión de caza (Bovy et al, en rev). De las seis especies encontradas, según la IUCN (53), dos se encuentran amenazadas: $L$. 
poeppigii (EN) y A. belzebuth (VU). Además, tres se encuentran también en el libro rojo de mamíferos del Ecuador (1). Esto es una razón más para continuar con el monitoreo de la comunidad de primates de la Reserva del Oglán Alto.

Basados en la cantidad de información recopilada, los grupos de primates que mayor número de avistajes presentaron fueron seleccionados como los grupos de estudio. Según Emmons (46), el área de vida de la especie $S$. fuscicollis, puede ser de 16 a 100 ha. El área de vida del grupo de este estudio (46,8 ha) es mayor a los estudios realizados en Bolivia $(15,25$ ha) y en Perú $(15,7$ a 16,5 ha) $(54,55)$. Hay que tomar en cuenta que estos estudios son realizados en Amazonía baja. El área de vida de $A$. seniculus (26,6 ha) es inferior a los estudios realizados en Caparú, Colombia (182 ha) y en Guyana (45 ha) pero levemente superior al estudio de La Macarena, Colombia (25 ha) $(56,57,58)$. Es importante destacar que las áreas de vida presentadas son propias para los grupos en estudio dentro de la reserva del Oglán Alto.

Durante el desarrollo de este estudio se registraron datos comportamentales, ocasionales, ya que en un $77,8 \%$ son comportamientos de vocalizaciones de alarma y eventos de escape, los cuales son característicos de grupos de primates no habituados, lo cual impide determinar las estrategias comportamentales de las especies en estudio (59).

En el Ecuador existen muy pocos estudios de primates en bosque de estribaciones amazónicas, por lo que estos resultados permitirán comparaciones y co-rrelaciones tan-to e c o lóg i c a s como etológicas con estudios de primates de la Amazonía baja y también del oc-

Figura 3. Curva Acumulación de Especies por jornadas de 3 meses de marzo 2007 a octubre 2008 
cidente del país.

\section{RECOMENDACIONES}

- Dadas las condiciones geográficas de los bosques de estribación, la preparación de un transecto lineal de más de un kilómetro y medio requiere un incremento no lineal del esfuerzo hombre-tiempo, y por lo tanto del dinero necesario. Por este motivo, se propone como forma de apoyo a esta metodología clásica el recorrido de senderos de una manera sistemática, previendo variables como picos de actividad y condiciones climáticas, además de un análisis de datos apropiado, lo que se cree es una alternativa para la determinación acertada de la densidad poblacional de primates.

- Es imprescindible mencionar que al monitorear a largo plazo estaríamos tomando constantemente datos demográficos sobre los grupos encontrados con el objetivo de no subestimar o sobrestimar la abundancia de primates del sector (Jost, comm. pers.), lo que aportaría confiabilidad a los análisis.

- No se debe realizar ningún análisis etológico mientras la habituación de los grupos no esté conseguida.

\section{AGRADECIMIENTOS}

Agradecemos a nuestras instituciones donantes FNRS, ECOCIENCIA, ORGÁNICA, AECID, UCE, así como a nuestros mentores y tutores Dr. Anthony Di-Fiore, Dr. Eduardo Fernández-Duque, Marie Claude Huynen; a nuestra institución Universidad Central del Ecuador, a los investigadores y profesores quienes nos han asesorado, Lou Jost, David Neill, Stella de la Torre, Esteban Suárez y un agradecimiento especial a la comunidad Pablo López del Oglán Alto, a todos los pasantes, voluntariados y tesistas del Proyecto Primates Ecuador.

\section{LITERATURA CITADA}

1. TIRIRA D. ed, 2001. Libro Rojo de los Mamíferos del Ecuador. QuitoEcuador.

2. CONSERVATION INTERNATIONAL, 2008. Estrategias de Conservación: Áreas Silvestres. PERU: CI PERU ed.

3. MITTERMEIER RA, RYLANDS $\mathrm{AB}, 2008$. The World's 25 most endangered primates - 2008-2010. In: IPS 2008. Edinburg.

4. SNETHLAGE E, 1910. Sobre a distribuicao da avifauna campestre na Amazônia. Boletim do Museu Emilio Goeldi 6:226-235.

5. SICK H, 1967. Rios e enchentes na Amazônia como obstáculo para a avifauna. Atas do Simpósio sobre a Biota Amazônica 5 (Zoologia): pp. 
495-520.

6. HAFFER J, 1969. Speciation in Amazonian forest birds. Science 165:131-137.

7. RYLANDS AB, 1987. Primate communities in Amazonian forests: Their habitats and food resources. Experientia 43:265-279.

8. CAPARELLA AP, 1988. Genetic variations in neotropical birds: implications for the speciation process. Acta Congressus Internationalis Ornithologici 19:1658-1673.

9. CAPARELLA AP, 1991. Neotropical avian diversity and riverine barriers. Acta Congressus Internationalis Ornithologici 20:307-316.

10. AYRES JM, CLUTTON-BROCK T, 1992. River boundaries and species range size in Amazonian primates. The American Naturalist 14:531-537.

11. HAFFER J, 1992. On the "river effect" in some forest birds of southern Amazonia. Boletim do Museu Paraense Emılio Goeldi Serie Zoologia 8:217-245.

12. CARDOSO DA SILVA JM, RYLANDS AB, DA FONSECA GAB, 2005. The Fate of the Amazonian Areas of Endemism. Conservation Biology 19:689-694.

13. GASCON C, BIERREGAARDM R, LAURANCE W, RANKIN-DEMERONA J, 2001. Deforestation and forest fragmentation in the Amazon. In: Lessons from Amazonia: the ecology and Conservation Biology (Jr. B,
Gascon C, Lovejoy T, Mesquita R, eds). New Haven, Connecticut: Yale University Press; 22-30.

14. CARRILLO-BILBAO GA, 2005. Primates ecuatorianos en Peligro, un Universo por Descubrir. El Gavilán Digital 5.

15. MITTERMEIER RA，CHENEY DL, 1987. Chapter 39: Conservation of primates and their habitats. In: Primate Societies (Smuts BB, Cheney DL, Seyfarth RM, Wrangham RW, Struhsaker TT, eds). Chicago: University of Chicago Press; pp. 477-490.

16. CHAPMAN CA, PERES CA, 2001. Primate Conservation in the New Millennium: The Role of Scientists. Evolutionary Anthropology 10:1633.

17. DE LA TORRE S, 2000. Primates de la Amazonía del Ecuador/ Primates of Amazonian Ecuador. In: Simbioe. Quito-Ecuador.

18. VAN ROOSMALEN M, VAN ROOSMALEN, T. Y MITTERMEIER, R.A., 2002. A taxonomic review of the titi monkeys, genus Callicebus Thomas, 1903, with the description of two new species, Callicebus bernhardi and Callicebus stephennashi, from Brazilia, Amazonia. Neotrop Primates 10(Suppl.):1-52.

19. ALBUJA V. L, 2002. Mamíferos del Ecuador. In: Diversidad y Conservación de los Mamíferos Neo- 
tropicales (Ceballos G, Simonetti JA, eds). México: CONABIO; 271-327.

20. CARRILLO-BILBAO GA, 2005. Uso del Tiempo, área de vida y especies vegetales que forman parte de la dieta de Callicebus discolor (Primates: Cebidae) en el Parque Nacional Yasuní. Tesis de Licenciatura. Quito: Universidad Central del Ecuador.

21. CARRILLO-BILBAO GA, MARTIN-SOLANO S, BRAVO J, HUYNEN M-C. eds, 2008. Ecuador and Belgium working for the conservation of primates. BELGIUM: Lieja University.

22. CHENEY DL, SEYFARTH RM, SMUTS BB, WRANGHAM RW, 1987. The Study of Primates Societies Research. In: Primate Societies (Smuts BB, Cheney DL, Seyfarth RM, Wrangham RW, Struhsaker TT, eds). Chicago: The University of Chicago Press; 1-8.

23. TIRIRA D, 1999. Mamíferos del Ecuador. Quito - Ecuador: ImpreFEPP.

24. DI FIORE A, 2004. Primate Conservation. New-York, EEUU.: MacGraw-Hill.

25. LOVETT JC, MARSHALL AR, 2006. Why should we conserve primates. African Journal of Ecology 44:113-115.

26. CHAPMAN CA, ONDERDONK DA, 1998. Forests without pri- mates: Primate/plant codependency. American Journal of Primatology 45:127-141.

27. COWLISHAW G, DUNBAR RIM, 2000. Chapter 4 - Community Ecology. In: Primates Conservation. Chicago, EUA: The University of the Chicago Press; pp. 57-92.

28. SIERRA R, 1999. Propuesta Preliminar de un Sistema de Clasificación de Vegetación para el Ecuador Continental. (INEFAN/GEF-BIRF P, ed). Quito- Ecuador: Ecociencia.

29. UNIVERSIDAD CENTRAL DEL ECUADOR, 2006. Plan de Manejo del Bosque del Oglán Alto. Arajuno-Pastaza. (Amazónica EC, ed). Quito-Ecuador: ECA.

30. CITES, 2000. Convención sobre el Comercio Internacional de Especies Amenazadas de Fauna y Flora Silvestre.

31. CERÓN CE, REYES C. MCA, VARGAS-GREFA M, 2006. La cuenca alta del río Oglán, PastazaEcuador, diversidad, ecología y flora. Quito: Editorial Universitaria.

32. OATES JF, 1987. Food distribution and foraging behavior. In: Primate societies (Smuts BB, Cheney DL, Seyfarth RM, Wrangham RW, Struhsaker TT, eds). Chicago: University of Chicago Press; pp 197-209.

33. REDFORD KH, ROBINSON JG, 1987. The game of choice: patterns of indian and colonist hunting in the 
neotropics. American Anthropologist 89:650-667.

34. BROWER J, ZAR, J., ENDE C. ed, 1998. Field and Laboratory Methods for General Ecology, 4th ed. USA: McGraw-Hill.

35. ROSS C, REEVE N, 2003. Survey and census methods: population distribution and density in: Setchell, J \& Curtis, D. (ed.). In: Field and Laboratory Methods in Primatology, a practical guide (Cambridge-University, ed). Cambridge-UK; pp. 91-101.

36. HIBY L, KRISHNA M, 2001. Line Transect Sampling from a Curving Path. Biometrics 57:727-731.

37. CARRILLO-BILBAO GA, 2004. Monitoreo Biológico, Socio-Económico y Cultural en el Bloque Sur. Reserva de la Biosfera Yasuní. Especialidad Primates. In: Monitoreo, Biológico, Socio-Económico y Cultural en el Bloque Sur Reserva de la Biosfera Yasuní (SIMBIOE, ed). Ecuador.

38. CARRILLO-BILBAO GA, 2005. Monitoreo Biológico, Socio-Económico y Cultural en el Bloque Tarapoa. Reserva de Producción Faunística Cuyabeno. Especialidad Primates. In: Monitoreo, Biológico, Socio-Económico y Cultural en el Bloque Tarapoa Reserva de Producción Faunística Cuyabeno (SIMBIOE, ed). Ecuador.

39. CARRILLO-BILBAO GA, DI FIORE A, FERNÁNDEZ-DUQUE E, 2005. Dieta, Forrajeo y Presupues- to de Tiempo en Cotoncillos (Callicebus discolor) del Parque Nacional Yasuní en la Amazonia Ecuatoriana. Neotropical Primates 13:7-11.

40. FERNANDEZ-DUQUE E, DI FIORE A, CARRILLO-BILBAO G, 2008. Behavior, Ecology, and Demography of Aotus vociferans in Yasuní National Park, Ecuador. Int J Primatol 29:421-431.

41. JOST L, 2006. Entropy and diversity. Oikos 113:363-375.

42. JOST L, 2007. Partitioning diversity into independent alpha and beta components. Ecology 88:2427 $-2439$.

43. JOST L, 2008. Dangerous diversity measures. In: Forum,Conservation Biology. Baños.

44. CROCKETT CM, EISENBERG JF, 1987. Howlers: Variations in Group Size and Demography. In: Primates Societies (Smuts BB, Cheney DL, Seyfarth RM, Wrangham RW, Struhsaker TT, eds). Chicago: The University of Chicago Press; 54-68.

45. WILSON GOLDIZEN A, 1987. Tamarins and Marmosets: Communal Care of Offspring. In: Primate Societies (Smuts BB, Cheney DL, Seyfarth RM, Wrangham RW, Struhsaker TT, eds). Chicago: The University of Chicago Press; 34-43.

46. EMMONS LH. ed, 1997. Neotropical Rainforest Mammals: A Field Guide., 2nd ed. IL: The University of Chicago Press, Chicago, 396 pp. 47. SCHMITT CA, DI FIORE A, LINK 
A, MATTHEWS LJ, MONTAGUE MJ, DERBY AM, HURST D, CARRILLO-BILBAO GA, SENDALL C, FIELD MY, FERNÁNDEZDUQUE E, 2007. Comparative ranging behavior of eight species of primates in a western Amazonian rainforest. Program of the SeventySixth Annual Meeting of the American Association of Physical Anthropologists.

48. TIRIRA D, BURNEO S, CASTRO I, 1996. Mamíferos del Parque Nacional Napo-Galeras (NapoEcuador). Quito: Jatun Sacha-Triton Ecuador.

49. CASTRO I, JACOME M, NOLIVOS L, SORNOZA F, 1999. Inventario de Fauna (Aves y Mamíferos) del Parque Nacional Sangay. Quito: Museo Ecuatoriano de Ciencias Naturales.

50. HEYMANN EW， ENCARNACIÓN C. F, 2002. Primates of the río Curaray, Northern Peruvian Amazon. International Journal of Primatology 23:191-201.

51. DI FIORE A, 2001. Investigación ecológica y de comportamiento de primates en el Parque Nacional Yasuní. In: Memorias del Seminario-Taller Yasuní: "Conservación y desarrollo sostenible del Parque Nacional Yasuní y su área de influencia“" - (Jorgenson JP, Coello Rodríguez M, eds). Quito, Ecuador: SIMBIOE; 165-173.

52. POZO R. WE, 2004. Habitat prefer- ences of six non-atelidae primates of Yasuni, Ecuador. Ecol apl 3:128-133.

53. IUCN, 2009. IUCN Red List of Threatened Species. Version 2009.2. <www.iucnredlist.org>. Downloaded on 22 January 2010.

54. SOINI P, 1987. Ecology of the Saddle-Back Tamarin Saguinus fuscicollis illiger on the Río Pacaya, Northeastern Peru. Folia Primatologica 49:11-32.

55. BUCHANAN-SMITH H， 1990. Polyspecific association of two tamarin species, Saguinus labiatus and Saguinus fuscicollis, in Bolivia. American Journal of Primatology 22:205-214.

56. IZAWA K, NISHIMURA A, 1988. Primate fauna at the study site, La Macarena, Colombia. Field Studies of New World Monkeys. La Macarena, Colombia 1:5-11.

57. JULLIOT C, 1992. Diet diversity and habitat of howler monkeys. XIVth congress of the International Primatological Society, Strasbourg, IPS:278.

58. PALACIOS E, RODRIGUEZ A, 2001. Ranging pattern and use of space in a group of red howler monkeys (Alouatta seniculus) in a southeastern Colombian rainforest. American Journal of Primatology 55:233-251.

59. WILLIAMSON EA, FEISTNER ATC, 2003. Habituating primates: processes, techniques, variables and ethics. In: Field and Laboratory 